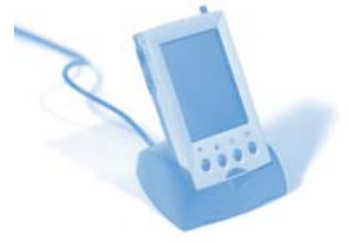

\author{
Jordan Balfour and \\ Antje Kohnle* \\ School of Physics and \\ Astronomy \\ University of St Andrews \\ St Andrews \\ KY16 9SS \\ *ak81@st-andrews.ac.uk
}

The importance of conceptual understanding

for problem-solving has

been demonstrated, in particular for problems requiring a transfer of knowledge to new contexts

\section{Testing conceptual understanding in introductory astronomy}

\begin{abstract}
Understanding students' prior beliefs about the nature of the Universe is a first step towards improving astronomy instruction. This article describes results from two diagnostic surveys testing understanding of astronomy concepts given to first, second and third-year St Andrews students taking astronomy and astrophysics modules. We highlight results pertaining to the phases of the Moon, the cause of the seasons, planet temperatures and properties of comets, and discuss possible underlying reasons for student difficulties. We find that some misconceptions remain at higher levels, and that new knowledge may be incorporated into prior beliefs without a substantial conceptual change.
\end{abstract}

\section{Introduction and Background}

Pre-existing student beliefs can strongly influence the development of new knowledge ${ }^{1}$. Knowing these pre-instructional beliefs that are common amongst students can allow the tailoring of instruction to promote substantial conceptual change. If these pre-instructional beliefs are inconsistent with current scientific understanding, they are often called misconceptions, and this term will be used in what follows. The importance of conceptual understanding for problem-solving has been demonstrated, in particular for problems requiring a transfer of knowledge to new contexts ${ }^{2}$. Standardised, validated multiple-choice diagnostic instruments to test conceptual understanding exist in many areas of physics, and also increasingly for astronomy and astrophysics (see also the article by S P Bates and R K Galloway in this volume). These instruments use incorrect choices (so-called distractors) that can elucidate common misconceptions. Examples for astronomy include the Astronomy Diagnostic Test ${ }^{3}$, the Astronomy and Space Science Concept Inventory ${ }^{4}$, the Light and Spectroscopy Concept Inventory ${ }^{5}$, the Lunar Phases Concept Inventory ${ }^{6}$ and the Star Properties Concept Inventory ${ }^{7}$. However, most of these astronomy inventories were created for School level or introductory university astronomy courses for non-science majors.

This work investigates understanding of astronomy concepts for physics and astrophysics students at the University of St Andrews. We describe the development of two surveys, one administered to level 1 (first-year) students in an introductory astronomy and astrophysics course, the other given to level 1, 2 and 3 (first, second and third year) students enrolled in astronomy and astrophysics modules. We describe selected results from these surveys, their implications and possible future work.

\footnotetext{
Methodology

In 2008/09 and 2009/10, students completed a diagnostic survey (called Solar System survey in what follows) covering topics from the Solar System part of the level 1 AS1001 course in the first and the last lecture of this course (pre- and post-test design). The survey consisted of nine multiple-choice questions on planet temperatures, phases of celestial objects, cause of the seasons, properties of comets, common features of satellites, and common properties of planets. Students were asked to choose one or more answers. An additional question asked about prior astronomy school courses. To analyse the survey results, we determined moments of the distribution of total scores and gains and normalised gains for each question and averaged over the survey ${ }^{8}$. The Solar System survey covered only one part of the AS1001 course, and students rated only their overall confidence, so that it was not possible to determine students' confidence in particular incorrect choices. This led us to develop a conceptual survey covering all parts of the AS1001 course, including 11 questions on the Solar System, 6 questions on stars and elementary astrophysics and 5 questions on galaxies and cosmology. The survey (called Astronomy Conceptual Survey, ACS, in what follows) was developed using known misconceptions from the literature and discussions with course lecturers and tutors. The survey was validated by trialling it with postgraduate astronomy
} 
students and staff. The survey was administered via WebCT (a virtual learning environment) as an anonymous survey to level 1, 2 and 3 students taking astronomy modules and to higher-level astronomy students on paper. Students were asked to choose one or more answers for each question, and to rate their certainty for each question.

To analyse the results, we determined moments of the distribution of total scores, performed t-tests to test for a significant difference in means between levels, and calculated effect sizes (defined as the difference in means divided by the average standard deviation) for significant differences. Due to small numbers, we grouped data from different levels where the distribution of responses was not significantly different. We determined discriminatory power of individual questions using the item difficulty index, item discrimination index and point biserial coefficient, and whole test reliability using the Kuder-Richardson reliability index and Ferguson's delta ${ }^{9}$, and found the values of these indicators to be satisfactory. Of particular interest were those questions that were answered with a high degree of certainty but a low percentage of correct answers.

\section{Student Survey outcomes}

For the Solar System survey, in 2008/09, 59 students took the pre- and 48 students took the post-test. In 2009/10, the numbers were 53 (pre-test) and 41 (post-test).

For the ACS, 22 of $49(45 \%)$ level 1 students, 17 of $22(77 \%)$ level 2 students and 21 of 28 (75\%) students in levels 3 and above completed the survey. Of those students at level 1 that completed the survey, 5 were astrophysics students, 15 are physics students and 2 are studying for other science degrees. At levels 2 and above, all students are studying for an astrophysics degree. The mean total score for the ACS (out of 22) was 12.9 for level 1 students, 13.4 for level 2 students and 15.6 for higher level students. The increase from level 1 to level 2 was not significant at the $95 \%$ confidence level, but the increases from level 2 to 3 (t-test, $p=0.044$, effect size $=0.70$ ), and level 1 to 3 (t-test, $p=0.013$, effect size $=0.81)$ were significant.

\section{Phases of the Moon}

Question 6 of the Solar System survey was as follows:

What causes the phases of the Moon?

A) Earth's shadow covering part of the Moon

$B$ ) The Moon's direction relative to the Sun as seen from Earth

It is easy to see that A) cannot be correct $^{10}$, as a gibbous Moon cannot be the result of Earth's shadow, and Sun and Moon would need to be on essentially opposite sides of the Earth all the time excepting for Full Moon, i.e., the Moon could not be in stable orbit around the Earth.

Figure 1 shows the results for this question both for the Solar System survey and the ACS. It is noticeable that a large number of students chose A) in the pre-test, namely 27 of 59 $(46 \%)$ in 2008/09 and 25 of $53(47 \%)$ in 2009/10. In the post-test, the fraction of students choosing the correct answer B) increased to $83 \%$ in $2008 / 09$ and $88 \%$ in $2009 / 10$, but in 2008/09 the fraction of students choosing the incorrect answer A) did not decrease accordingly: while one student chose both answers in the pre-test, seven students chose both answers in the post-test. In 2009/10, 4 of 41 students chose both answers in the post-test. Although these are small student numbers, these results may show that instruction does not necessarily lead to the revision of prior beliefs, but instead may lead to an inconsistent set of ideas. It would seem that misconceptions need to be explicitly challenged in order for students to

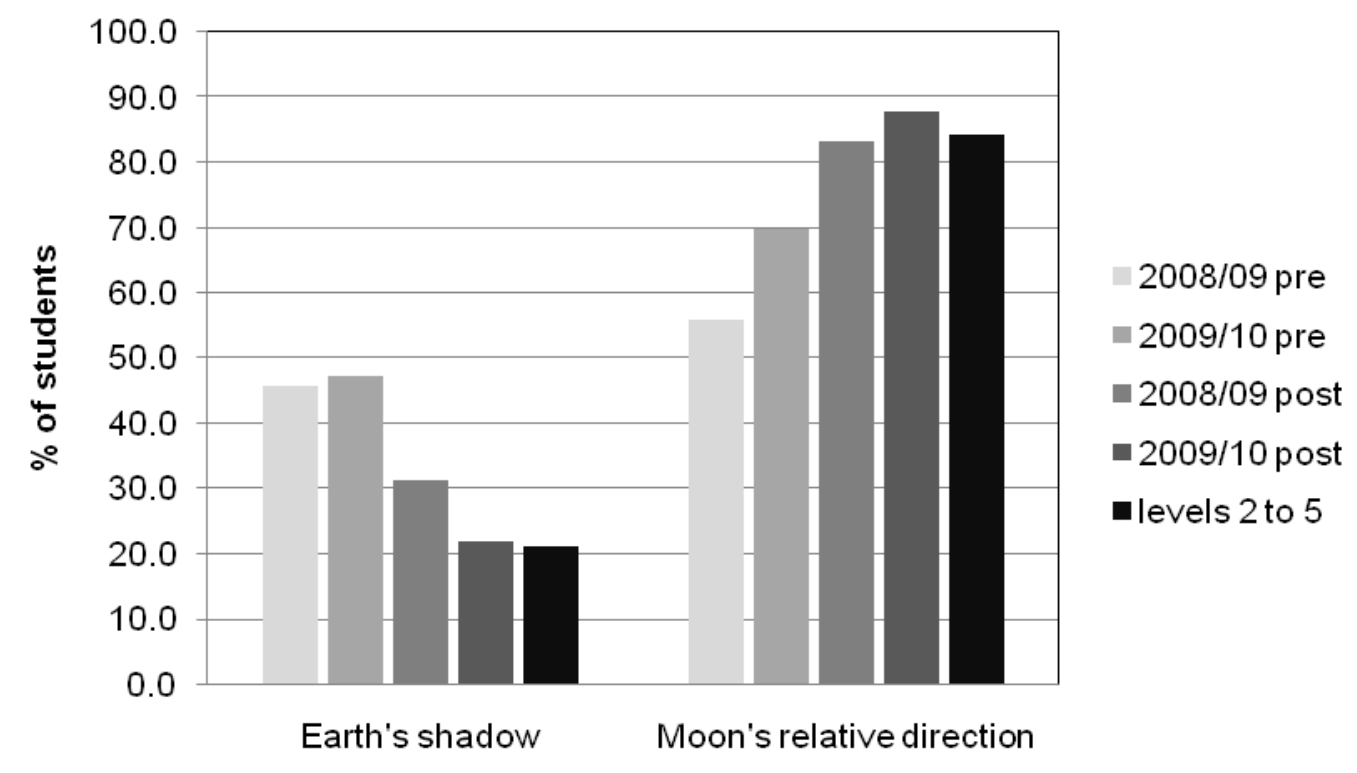

Figure 1: The figure shows the responses to the question "What causes the phases of the Moon?" (see text for full details of choices) for level 1 introductory astronomy students pre- and post-instruction for two different years, as well as for astrophysics students in levels 2 to 5 from a survey conducted in Spring 2010. The level 1 course is taken by physics, astrophysics and science students, whereas the level 2 and above courses are only taken by astrophysics students. The total student numbers surveyed range from 38 (levels 2 and above) to 59 (2008/09 pre). 
overcome them, in particular when these prior beliefs are deep-rooted in everyday experience. The ACS contained a slightly revised version of the above question (including an additional choice $\mathrm{C}$ ), allowing comparisons with astrophysics students in levels 2 and above (see Figure 1 ). $21 \%$ ( 8 of 38 ) of higher-level students stated choice $A$ is correct, two students in this group stated that both $A$ ) and B) are correct. All these students were certain of their answer, excepting one student who stated "somewhat certain".

\section{Cause of the seasons}

Question 1 of the ACS was as follows:

Which of the following predominantly causes the seasons on Earth?
A) The changing distance from the Earth to the Sun
$B$ ) The tilt of the Earth's axis causing one
hemisphere to be closer to the Sun than the other
C) The tilt of the Earth's axis inducing changes in sunlight intensity and day length

While there is a difference in temperatures due to the axis tilt (choice $\mathrm{B}$ ), it is exceedingly small $(0.02 \mathrm{~K})$, so cannot be the cause of the seasons. Figure 2 shows the results. No students chose response A), which is not shown in the figure. Results were similar for level 1, 2 and 3 students, leading us to sum the data for all levels. No student chose "not at all confident" as confidence rating. $37 \%$ of students chose $B$ ). The confidence of students choosing B) was quite high, but lower than for the correct response $\mathrm{C}$ ).

These results coincide with the results from the AS1001 exam in 2010: almost all students were able to calculate correctly the difference in equilibrium temperatures at Earth's perihelion and aphelion (the points nearest and furthest away from the Sun on its elliptical orbit), 4K for a difference in distance of 5 million $\mathrm{km}$. In contradiction to this, 9 of 46 students (19.6\%) stated in the exam that the seasons are caused by the hemispheres alternately being closer to the Sun, with all but one of these students getting full marks or almost full marks on the previous calculation.

How can one explain these results? Comins ${ }^{10}$ argues that over-generalisation of a general principle based on our everyday experience may be an underlying cause: in this case, the principle would be that the closer we are to a heat source, the more heat we feel. This would agree with the level 1 results that pre-instruction, $54 \%$ of students state that Mercury is the hottest planet, not Venus.

When asked about common properties of all known Solar System satellites, post-instruction $32 \%$ of level 1 to 3 students combined (81 students in total) state 'they are smaller than the smallest planet' (Ganymede is larger than Mercury), 20\% state 'they are practically spherical' (e.g., Mars' satellites aren't), and $9 \%$ state 'they have no atmosphere' (Titan does). Here, the underlying cause may be the overgeneralisation of the properties of our Moon. Another underlying principle may be the belief in permanence of celestial objects: In a question on comet properties, pre-instruction only $31 \%$ of level 1 students agreed with the statement "Comets disintegrate after 100 to 200 passages close to the Sun" (2008/09 and 2009/10 data combined). Post-instruction, this number rose to $79 \%$.

\section{Discussion and outlook}

Our results show that some misconceptions in astronomy persist to higher levels, and are not easily corrected. They also illustrate the importance of stressing conceptual understanding in astronomy instruction, and getting students to relate results of calculations to astronomy concepts.

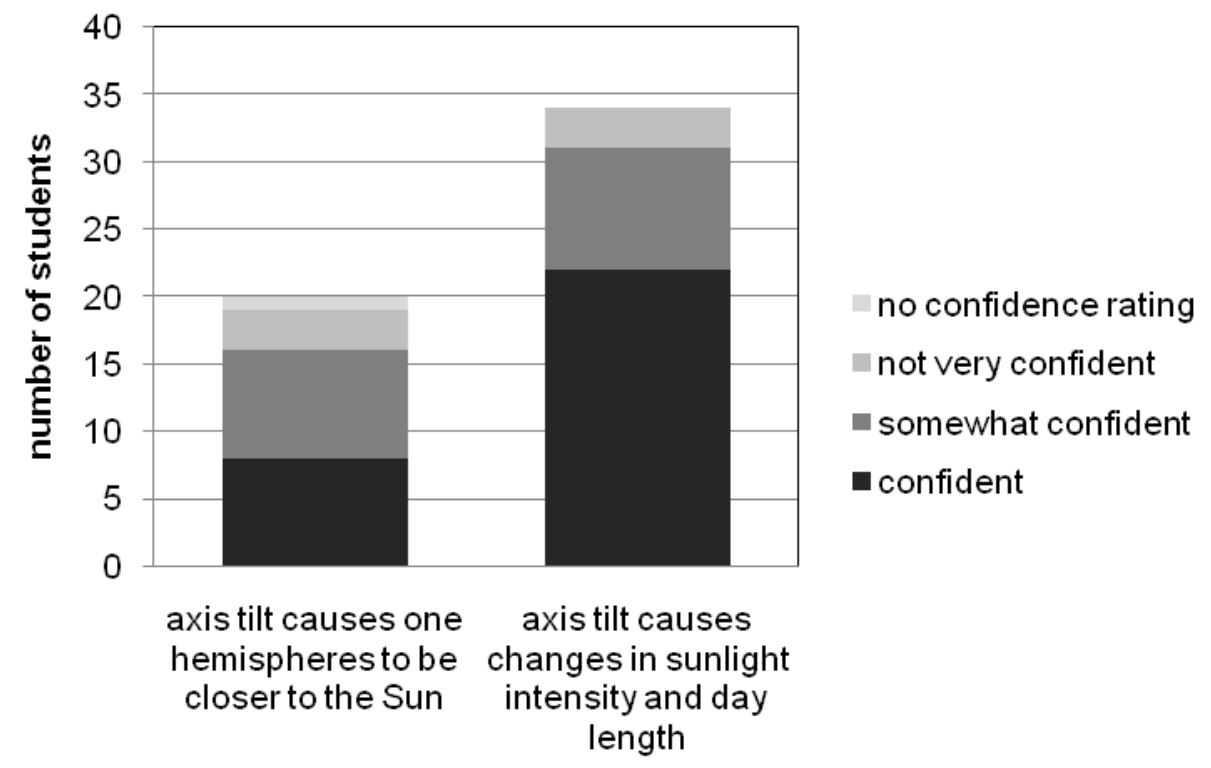

Figure 2: The figure shows the responses and confidence ratings to the question "Which of the following predominantly causes the seasons on Earth?" (see text for full details of choices) for students taking astronomy modules in levels 1 to 3. The data include 22 level 1, 17 level 2 and 15 level 3 students. 
We found that the distribution of responses to the ACS questions was similar across the levels, and that the increase in average score with increasing level was only modest. This may be due to the fact that higher-level astronomy courses do not stress the concepts tested in the ACS in detail.

Limitations of this work are relatively small student numbers, lack of free text explanations of reasoning or student interviews to uncover underlying reasons for incorrect choices, and the fact that this investigation was only carried out at a single institution. Knowing students' astronomy misconceptions can only be a first step in the ultimate aim of helping students to come to a correct understanding of the nature of the Universe. Future work includes extending this study to other institutions, gaining more insight into underlying reasons for misconceptions in astronomy, and developing and evaluating course material to target specific misconceptions.

\section{References:}

1. Halloun, A. and Hestenes, D. (1985) The initial knowledge state of college physics students, American Journal of Physics, 53 (11), 1043-1055.

2. Mazur, E. (1997) Peer instruction, New Jersey, Prentice Hall.

3. Zeilik, M (2002) Birth of the Astronomy Diagnostic Test: Prototest Evolution, Astronomy Education Review, 1 (2), 46-52.

4. Sadler, P.M, Coyle, H., Jaimie, L., Cook-Smith, N., Dussault, M., Gould, R.R. (2009) The Astronomy and Space Science Concept Inventory: Development and Validation of Assessment Instruments Aligned with the K-12 National Science Standards, Astronomy Education Review, 8 (1), 010111.

5. Bardar, E.M., Prather, E.E., Brecher, K. and Slater, T.F. (2006) Development and Validation of the Light and Spectroscopy Concept Inventory, Astronomy Education Review, 5, (2), 103-113.

6. Lindell, R. and Olsen, J. (2002) Developing the Lunar Phases Concept Inventory, Proceedings of the 2002 Physics Education Research Conference, Franklin, S., Marx, J., and Cummings, K. (Editors), New York, PERC Publishing.

7. Bailey, J.M. (2008) Development of a Concept Inventory to Assess Students' Understanding and Reasoning Difficulties about the Properties and Formation of Stars, Astronomy Education Review, 6 (2), 133-139.

8. Hake, R.R. (1998) Interactive-engagement versus traditional methods: A six-thousand-student survey of mechanics test data for introductory physics courses, American Journal of Physics, 66 (1), 64-74.

9. Ding, L., Chabay, R., Sherwood, B., and Beichner, R. (2006) Evaluating an electricity and magnetism assessment tool: Brief electricity and magnetism assessment, Phys. Rev. ST Phys. Educ. Res., 2, (1), 010105.

10. Comins, N.F. (2001) Heavenly Errors: Misconceptions about the Real Nature of the Universe, New York, Columbia University Press.

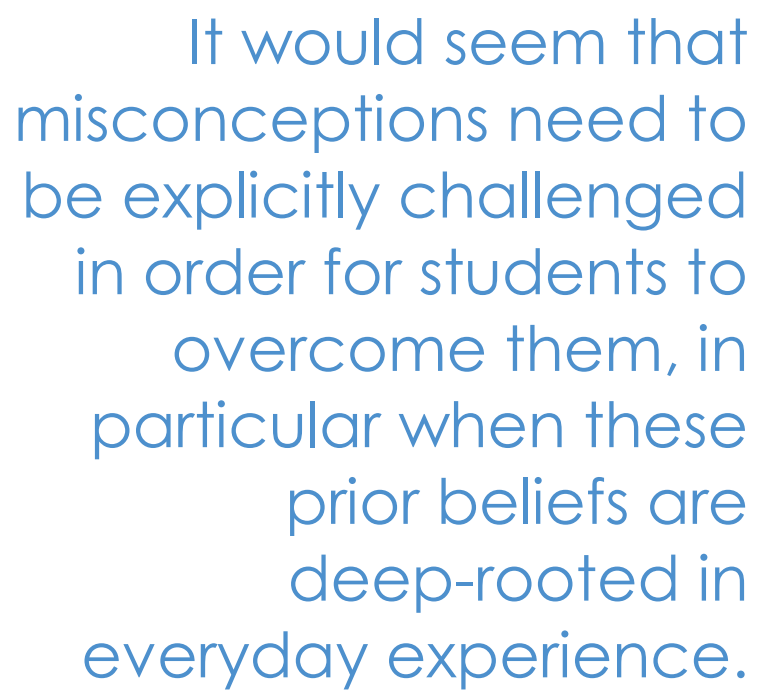

It would seem that misconceptions need to explicitly challenged in order for students to overcome them, in prior beliefs are deep-rooted in everyday experience. 